\title{
CCR6 Is a Predicting Biomarker of Radiosensitivity and Potential Target of Radiosensitization in Rectal Cancer
}

Hui Chang, MD',2
Jia-wang Wei, MD
Ya-lan Tao, MD',
Pei-rong Ding, MD
Yun-fei Xia, MD',
Yuan-hong Gao, MD
Wei-wei Xiao, MD

${ }^{1}$ State Key Laboratory of Oncology in South China, Collaborative Innovation Center for Cancer Medicine, Guangzhou,

Departments of ${ }^{2}$ Radiation Oncology and ${ }^{3}$ Colorectal Surgery, Sun Yat-sen University Cancer Center, Guangzhou, China
Correspondence: Yuan-hong Gao, MD Department of Radiation Oncology, Sun Yat-sen University Cancer Center, 651 Dongfeng Road East, Guangzhou, Guangdong 510060, China

Tel: 86-13560182168

Fax: 86-020-87343491

E-mail: gaoyh1962@126.com

Co-correspondence: Wei-wei Xiao, MD Department of Radiation Oncology, Sun Yat-sen University Cancer Center, 651 Dongfeng Road East, Guangzhou, Guangdong 510060, China

Tel: 86-13710390520

Fax: 86-020-87343491

E-mail: xiaoww@sysucc.org.cn

Received November 11, 2017

Accepted December 20, 2017

Published Online December 21, 2017

*Hui Chang and Jia-wang Wei contributed equally to this work.

\section{Purpose}

This study aimed to explore the functions and mechanisms of $\mathrm{C}-\mathrm{C}$ motif chemokine receptor 6 (CCR6), a gene associated with progression and metastasis of colorectal cancer (CRC), in radiosensitivity of rectal cancer $(\mathrm{RC})$.

\section{Materials and Methods}

RNA sequencing and immunohistochemical analysis on CCR6 expression were performed in pretreatment tissues of RC patients exhibiting different therapeutic effects of radiotherapy. Colonogenic survival assay was conducted in different CRC cell lines to assess their radiosensitivity. And the impact of CCR6 expression on radiosensitivity was validated through RNA interference. The DNA damage repair (DDR) abilities of cell lines with different CCR6 expression were evaluated through immunofluorescence-based $\mathrm{yH} 2 \mathrm{AX}$ quantification.

\section{Results}

The CCR6 mRNA level was higher in patients without pathologic complete remission (pCR) than in those with pCR (fold changed, 2.11; $p=0.004$ ). High-level expression of CCR6 protein was more common in the bad responders than in the good responders $(76.3 \% \mathrm{vs.} 37.5 \%$, $p<0.001$ ). The CRC cell lines with higher CCR6 expression (LoVo and sw480) appeared to be more radioresistant, compared with the sw620 cell line which had lower CCR6 expression. CCR6 knockdown made the LoVo cells more sensitive to ionizing radiation (sensitization enhancement ratio, 1.738; $p<0.001$ ), and decreased their DDR efficiency.

\section{Conclusion}

CCR6 might affect the RC radiosensitivity through DDR process. These findings supported CCR6 as a predicting biomarker of radiosensitivity and a potential target of radiosensitization for RC patients. 


\section{Introduction}

Colorectal cancer (CRC), especially rectal cancer ( $R C)$, is one of the most common malignancies in China [1]. The presurgical radiotherapy (RT) is now a necessary constituent of the standard treating mode for locally advanced RC [2]. The tumor regression grade (TRG) after RT has been proven as an independent factor predicting the long-term survival of the patients [3]. However, even after standard chemoradiotherapy (CRT), only $21.3 \%$ of the patients attained pathologic complete remission ( $\mathrm{pCR}$ ). And the good responders occupy merely $31.2 \%$ of the cases [4]. To achieve success of $\mathrm{RT}$ and improve prognosis, cellular resistance to ionizing radiation (IR) remains the primary barrier to overcome.

DNA damage repair (DDR) is acknowledged as the most classical mechanism of cellular radioresistance [5]. And recent studies revealed that tumor microenvironment (TME) also played important roles in radiosensitivity modulation [6]. Chemokine receptors, a kind of $G$ protein-coupled transmembrane molecules regulating organogenesis and inflammation under physiological state, are now known as bridges through which the TME impacts many biological procedures within the tumor cells [7]. The C-C motif chemokine receptor 6 (CCR6) is the most-studied chemokine receptor in CRC. It is overexpressed abnormally in CRC cells and the surrounding tumor-associated immune cells. And a negative association have been reported between CCR6 expression and the patients' clinical outcome $[8,9]$. The CCR6 has also been demonstrated to promote oncogenesis, progression, and metastasis of CRC $[9,10]$. Yet, there is no evidence on the functions of CCR6 in RC radioresistance until now. Since the downstream signaling molecules of CCR6, the Akt and the ERK, could regulate DDR through enhancing the stability of the direct participants, such as DNA-PK and Rad51 [11-14], we hypothesized that CCR6 might influence radiosensitivity of $\mathrm{RC}$, and be a new target for radiosensitization therapy. In this study, we determined the relationship between CCR6 expression and $\mathrm{RC}$ radioresistance, through patients and cell lines exhibiting different response to IR. The modulating ability of CCR6 on radiosensitivity was further confirmed by CCR6 knockdown.

\section{Materials and Methods}

\section{Tissue specimens}

The pretreatment tumor tissue of the patients who had pathologically diagnosed $\mathrm{RC}$ and received CRT was from the tumor bank of the Sun Yat-sen University Cancer Center (SYSUCC). The paraffin-embedded specimens were for immunohistochemical (IHC) staining, and the specimens contained in RNAlater (Thermo Fisher Scientific, Waltham, MA) were for RNA sequencing (RNA-seq).

\section{Evaluation of TRG}

The postoperative pathology of each patient treated with radical resection was assessed by an experienced attending pathologist. The TRG was determined according to the Mandard standard [4], in which PCR was defined as TRG 1.

\section{RNA-seq}

Among the patients receiving surgery between May 1, 2013 and August 31, 2013, six cases were randomly selected from those exhibiting $\mathrm{pCR}$ in the postsurgical pathology. And another six cases were randomly selected from those exhibiting non-pCR. RNA-seq was performed for these 12 patients, using the HiSeq 4000 system (Illumina, San Diego, CA). The pathoclinical profiles of the patients were shown in S1 Table (see Supplementary Materials).

The expression level was represented with fragments per kilobase million (FPKM) [15]. The fold change (FC) of each gene was calculated through dividing the average FPKM of the non-pCR group by that of the $\mathrm{pCR}$ group. The difference in the average FPKM between the two groups was tested by a student's $t$ test. Hierarchical clustering analysis based on Euclidean distance was performed in genes with a p-value of $<0.01$, through MultiExperiment Viewer 4.9.0 (available at http://tm4.org). And, pathway enrichment analysis was also made in those genes through Metascape [16].

\section{IHC analysis}

IHC analysis was performed in the consecutive patients who received surgery between November 1, 2015 and September 30, 2017. Before staining, all the paraffin-embedded specimens were deparaffinized with xylenes, rehydrated with graded ethanol to distilled water, and submerged in EDTA antigen retrieval buffer $(1 \mathrm{mmol} / \mathrm{L}, \mathrm{pH} 8.0)$ and microwaved to retrieve antigens. IHC staining was then performed using the Dako REAL Envision system, Peroxidase/DAB, Rabbit/Mouse (Dako, Carpinteria, CA), following the manufacturer's recommended protocols. After treatment with $0.3 \%$ hydrogen peroxide for 15 minutes and normal goat serum for 30 minutes, the specimens were incubated with a CCR6 rabbit polyclonal antibody (1:100, ab109703, Abcam, Cambridge, UK) overnight at $4^{\circ} \mathrm{C}$. The sections were then incubated with a peroxidase-conjugated Dako REAL EnVision/HRP, Rabbit/Mouse (ENV) reagent 
(solution A) for 30 minutes. Finally, the visualization was done by incubating the sections in DAKO REAL DAB+ Chromogen (solution C) for 10 minutes. The slides were washed with phosphate buffered solution (PBS) between the staining steps.

The expression of CCR6 was evaluated by another experienced attending pathologist who was blinded to the TRG of the patients. The entire tissue section was observed to assign scores of intensity and extent. The intensity scores included as 0 (no staining), 1 (light yellow), 2 (yellow brown), and 3 (brown). The extent scores were decided according to the percentages of the positive cells in the whole carcinoma area, included $0(0 \%), 1(1-25 \%), 2(26-50 \%), 3(51-75 \%)$, and 4 (76-100\%). The final staining score (0 to 12$)$ was the product of the intensity and extent scores. Tumors with a staining score of $<4$ were defined as low expression, and those with a score $\geq 4$ were defined as high expression (S2 Fig.).

The median IHC staining scores of the resistant group (patients with TRG 3-5) and the sensitive group (patients with TRG 1-2) were compared through a Mann-Whitney U test. And proportions of the cases with high CCR6 expression in these two groups were also compared, through a chisquare test.

\section{Cell culture}

The CRC cell lines used in this study included LoVo, sw480 and sw620, which were purchased from the American Type Culture Collection (Manassas, VA) and maintained in the SYSUCC [17]. These cells were all cultured in Roswell Park Memorial Institute 1640 medium (Thermo Fisher Scientific) supplemented with $10 \%$ fetal bovine serum (Thermo Fisher Scientific), penicillin (100 units $/ \mathrm{mL}$ ), and streptomycin (100 units / mL). An incubator was used to create a humidified atmosphere of $5 \% \mathrm{CO}_{2}$ and a constant temperature of $37^{\circ} \mathrm{C}$ for cell culture.

\section{RNA interference}

To knockdown the CCR6 expression, transient transfection of small interfering RNA (siRNA) was performed, using the Lipofectamine 3000 Transfection Reagent (Thermo Fisher Scientific). The siRNA duplexes were synthesized by the Genechem Co. (Shanghai, China), and consisted of $5^{\prime}$-GGUCUAUGACAGACGUCUAUCdTdT- $3^{\prime}$ and 5'-UAGACGUCUGUCAUAGACCUGdTdT-3' as the sense and antisense sequences, respectively. The AllStars Negative Control siRNA (Qiagen, Hilden, Germany) was used as the negative control. The cells were harvested 24 hours after transfection for the subsequent experiments.

\section{Irradiation and clonogenic survival assay}

The cells were trypsinized and seeded into 6-well plates. Different cell quantities were for different doses of irradiation $\left(100,200,10^{3}\right.$, and $10^{4}$ cells were for $0,2,4$, and $6 \mathrm{~Gy}$, respectively).The irradiation was performed by a R2000 X-ray irradiator (Rad Source Technologies, Suwanee, GA), with a dose rate of $1.1 \mathrm{~Gy} / \mathrm{min}$, a voltage of $160 \mathrm{kV}$, a current of $25 \mathrm{~mA}$, and 0.3-mm copper filters. The cells were then cultured as we described above for 14 days, fixed by $4 \%$ paraformaldehyde, and stained with Giemsa. Colonies with more than 50 cells were scored as survivors. The plating efficiency (PE) was calculated by dividing the number of survivors by the number of plated cells at $0 \mathrm{~Gy}$. Surviving fraction (SF) at each dose was determined as the number of survivors divided by the product of the PE and the number of plated cells at the corresponding dose. Sensitization enhancement ratio (SER) was calculated by division of SF at 2 Gy (SF2). Clonogenic survival curves were fitted to the linear quadratic model, $S F=\exp \left[-\left(\alpha \mathrm{D}+\beta \mathrm{D}^{2}\right)\right]$, and compared through the extra-sum-of-squares $F$ test.

\section{Western blot analysis}

The expression level of CCR6 protein in CRC cells, including those transfected with siRNA, was examined by western blot analysis. The procedure of western blot referred to a report of Cerda et al. [18] prior to our study. After lyses with the RIPA buffer, the total protein in the cells were collected at $4^{\circ} \mathrm{C}$ and quantified by a Micro BCA Protein Assay Kit (Thermo Fisher Scientific). Protein of $50 \mu \mathrm{g}$ was mixed with sample buffer, which consisted of $250 \mathrm{mmol} / \mathrm{L}$ Tris- $\mathrm{HCl}(\mathrm{pH}$ 6.8), $8 \%$ sodium dodecyl sulfate (SDS), $0.04 \%$ bromophenol blue, $40 \%$ glycerol, and $20 \% \beta$-mercaptoethanol. The mixture was heated for 5 minutes at $95^{\circ} \mathrm{C}$, resolved by SDS polyacrylamide gel electrophoresis (the concentration of polyacrylamide gel was 10\%), and transferred to nitrocellulose membranes (Thermo Fisher Scientific) by electroblotting. The membranes were blotted with $5 \%$ skimmed milk, washed with Tris-buffered saline Tween, and incubated with the primary anti-CCR6 rabbit polyclonal antibody (1:1,000, ab109703 , Abcam) overnight at $4^{\circ} \mathrm{C}$. After washing, the membranes were incubated with anti-rabbit horseradish peroxidase conjugated IgG (1:2,000, Santa Cruz Biotechnology, Santa Cruz, CA) for 1 hour. Fluorescence detection was then performed through the enhanced chemiluminescence (Amersham Pharmacia Biotech, Piscataway, NJ). Glyceraldehyde 3-phosphate dehydrogenase (GAPDH) was used as a loading control. The integrated option density (IOD) of a specific band was measured by Image-pro Plus 6.0 (Media Cybernetics, Rockville, $\mathrm{MD})$. The CCR6 expression level in each cell line was represented with the IOD of the CCR6 band divided by the IOD 
of the GAPDH band. The FC was calculated by dividing the CCR6 expression level in a specific cell line by that in the reference cell line.

\section{Reverse transcription-polymerase chain reaction}

The expression level of CCR6 mRNA in CRC cells was examined by reverse transcription-polymerase chain reaction (RT-PCR) analysis. Total RNA from different CRC cell lines were extracted with Trizol reagent (Thermo Fisher Scientific). Then the first-strand cDNA was synthesized with $1 \mu \mathrm{g}$ of total RNA, through the SuperScript III First-Strand Synthesis System (Thermo Fisher Scientific). Realtime RT-PCR was performed in triplicate for each sample, using the Absolute qPCR SYBR Green Mixes (Thermo Fisher Scientific). PCR reaction and data collection were done through the ABI PRISM7900HT sequence detection system (Thermo Fisher Scientific). GAPDH was used as the endogenous control for normalization. The primer sequences for CCR6 were 5'-ACCGCAGATAACGACAATGC-3' (sense) and 5'-CATGAGCACGTTAAGTCCCG-3' (anti-sense), which were designed by the Primer3 4.1.0 [19]. And the primer sequences for GAPDH were 5'-CTCCTCCTGTTCGACAGTCAGC-3' (sense) and 5'-CCCAATACGACCAAATCCGTT-3' (antisense) [20].

\section{Immunofluorescence analysis}

Immunofluorescence (IF) staining countering phosphorylated histone $\mathrm{H} 2 \mathrm{AX}(\gamma \mathrm{H} 2 \mathrm{AX})$ was made to evaluate the DDR abilities of CRC cells [20]. First, the cells were seeded on coverglasses in 24-well plates $\left(5 \times 10^{4}\right.$ cells per well), cultured for 24 hours, and irradiated with an X-ray of $2 \mathrm{~Gy}$. At 30 minutes and 24 hours after irradiation, the cells were fixed (4\% paraformaldehyde, 15 minutes) and permeabilized (0.25\% Triton-X 100 in PBS, 15 minutes) at $4^{\circ} \mathrm{C}$. Blocking was carried out by adding $5 \%$ bovine serum albumin in PBS for $30 \mathrm{~min}-$ utes. Next, the cells were incubated with an anti- $\gamma \mathrm{H} 2 \mathrm{AX}$ (Ser139) rabbit polyclonal antibody (1:1,000, ab2893, Abcam) for 2 hours, followed by Alexa 488-conjugated goat anti-rabbit IgG (1:500, ab150077, Abcam) for 1 hour. The nuclei were counter stained with $1 \mu \mathrm{g} / \mathrm{mL}$ DAPI solution (Thermo Fisher Scientific). Finally, coverslips were mounted with Vectashield (Vector Laboratories, Peterborough, UK). Images were then taken through an Olympus FV100 confocal imaging system (Olympus Co., Tokyo, Japan). At each time point, IF staining was conducted in triplicate for each cell line. Difference in average number of the $\gamma \mathrm{H} 2 \mathrm{AX}$-positive cells between any two cell lines at a defined time point were compared by a student's t test.

\section{Statistical analysis}

Statistical analysis in this study was executed by IBM SPSS Statistics ver. 23.0 (IBM Co., Armonk, NY) or GraphPad Prism 5.0 (GraphPad Software, La Jolla, CA) if not special specified. A two-sided $\mathrm{p}$-value of $<0.05$ was considered statistically significant.

\section{Ethical statement}

The use of tissue specimens was approved by the Institutional Review Board of the SYSUCC (approval No. GZR2017079). And written informed consent was obtained from all patients whose specimens were involved in this study.

\section{Results}

\section{CCR6 expression was inversely correlated with radiosen- sitivity of RC patients}

To determine how CCR6 influenced the treatment effects of RT, we first assessed the expression level of CCR6 mRNA through RNA-seq in RC patients exhibiting different response to RT. Among the 34,316 genes sequenced, there were totally 22,978 genes presenting expression. Of those, differential expression $(p<0.05)$ was seen in 768 genes, in which 116 genes exhibited obviously differential expression $(\mathrm{p}<$ 0.01), including the CCR6 gene. The volcano plot and the clustering heatmap of these 116 genes were shown as Fig. 1A and $\mathrm{B}$, respectively. The average FKPM of CCR6 gene in the non-pCR group was greater than that in the $\mathrm{pCR}$ group (FC, 2.11; $\mathrm{p}=0.004)$. Hence, the CCR6 mRNA was expressed more highly in the non-pCR patients than in the extremely good $(\mathrm{pCR})$ responders. Additionally, the chemokine-mediated signaling pathway in which CCR6 took part was one of the top 5 enriched pathways (Fig. 1C).

To confirm the results of RNA-seq, we performed evaluation on expression level of CCR6 protein in an enlarged sample of patients. There were a total of 95 patients undergoing IHC analysis, including 55 cases in the resistant (TRG 3-5) group and 40 patients in the sensitive (TRG 1-2) group. Except CCR6 expression, the pathoclinical profiles of the patients were balanced between the two groups (S3 Table). The median score of the resistant group was greater than that of the sensitive group ( 8 vs. 3, $\mathrm{p}=0.005$ ) (Fig. $2 \mathrm{~A}$ ). And highlevel CCR6 expression was more common in the resistant group than in the sensitive group $(76.3 \%$ vs. $37.5 \%, \mathrm{p}<0.001)$ (Fig. 2B). The results indicated that CCR6 protein was expressed more highly in the bad responders than in the 
$\infty$

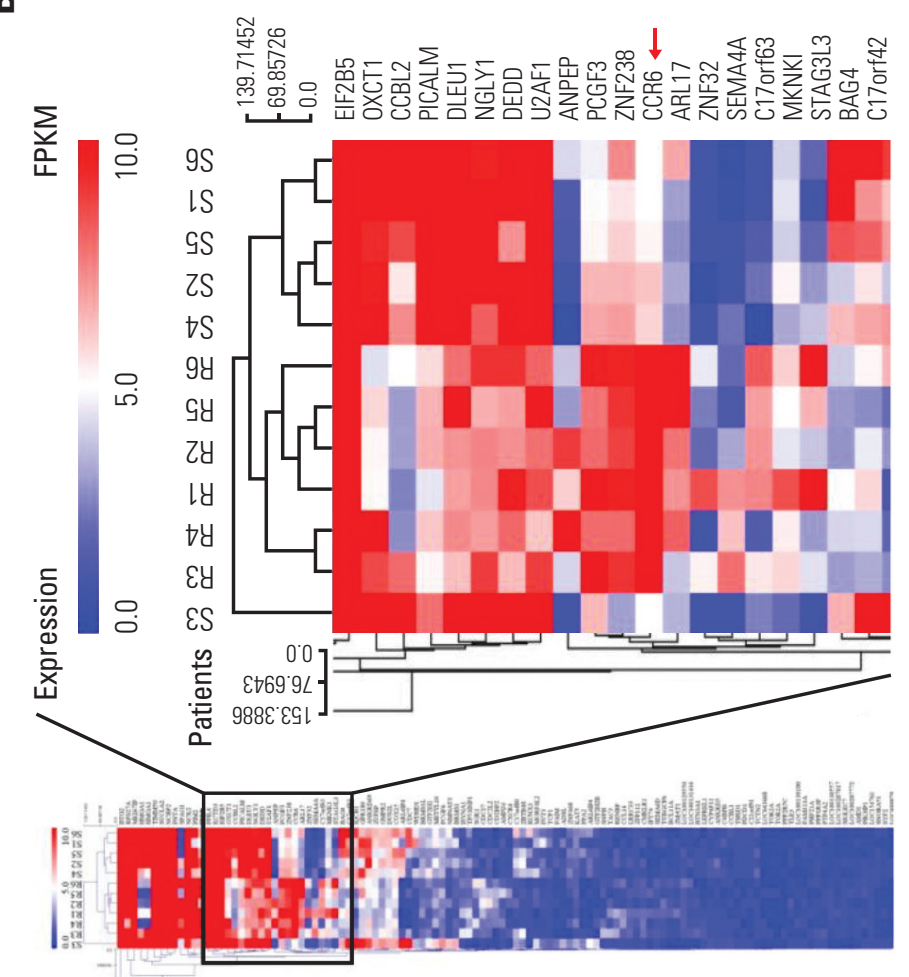

플

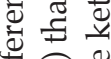

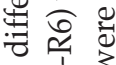

몬

.

它

bo 2

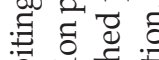

를

ช

$\mathscr{2}: 1010$

कृ की

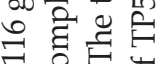

चु

I $\frac{\dot{9}}{\infty}$

넝응 중

르류 졸

छ

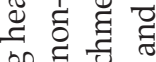

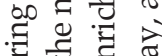

들 己⿱艹

㐘

ปั

๑ิ

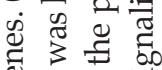

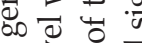

రृ

के

त्र

$\infty$ 网

ते

\&

¿

$\circ$ ह हो

흘 흄등

$\circ$ 의

ส

웡

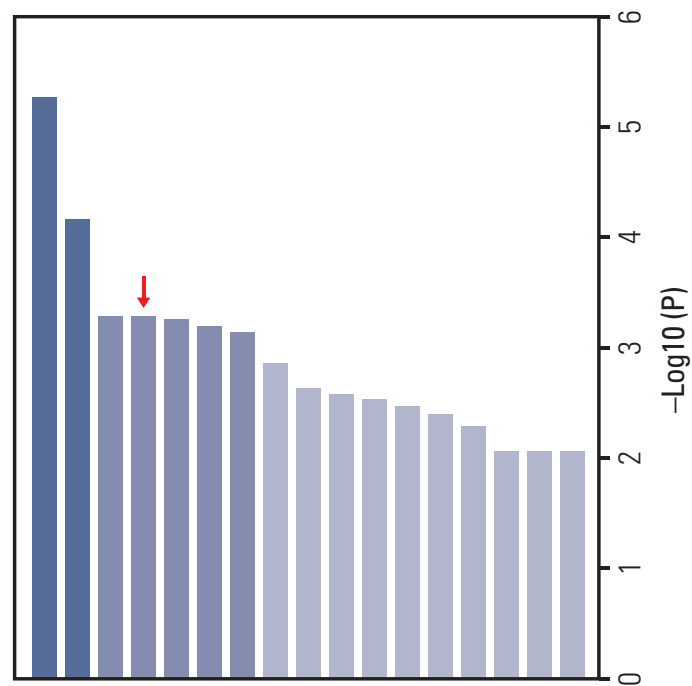

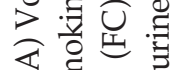

¿ व

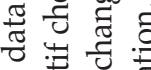

bo च च

छ

U บ

过它 导

क

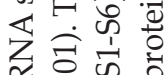

पै

$\stackrel{8}{*}$.

듕 을

I1.

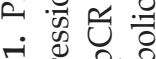

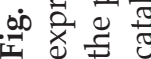




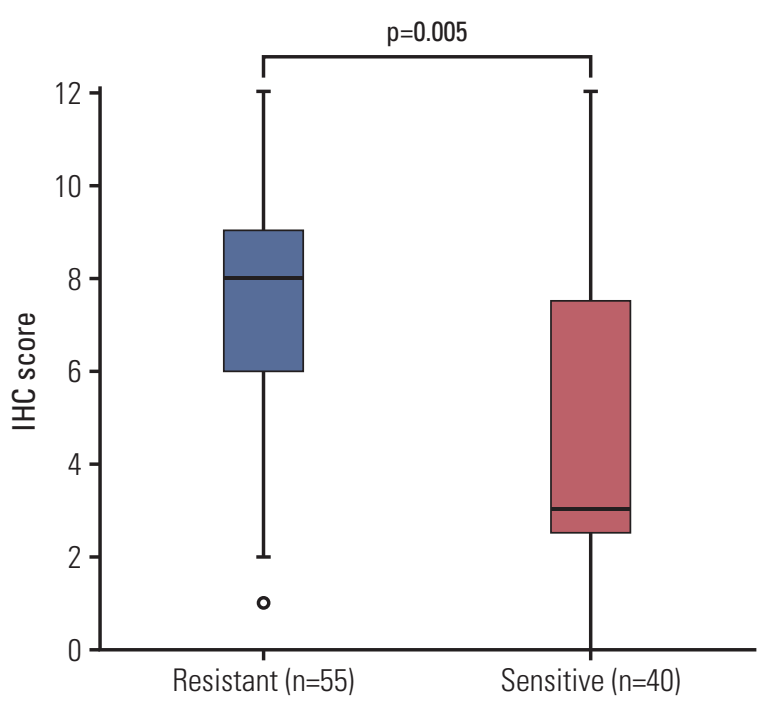

A

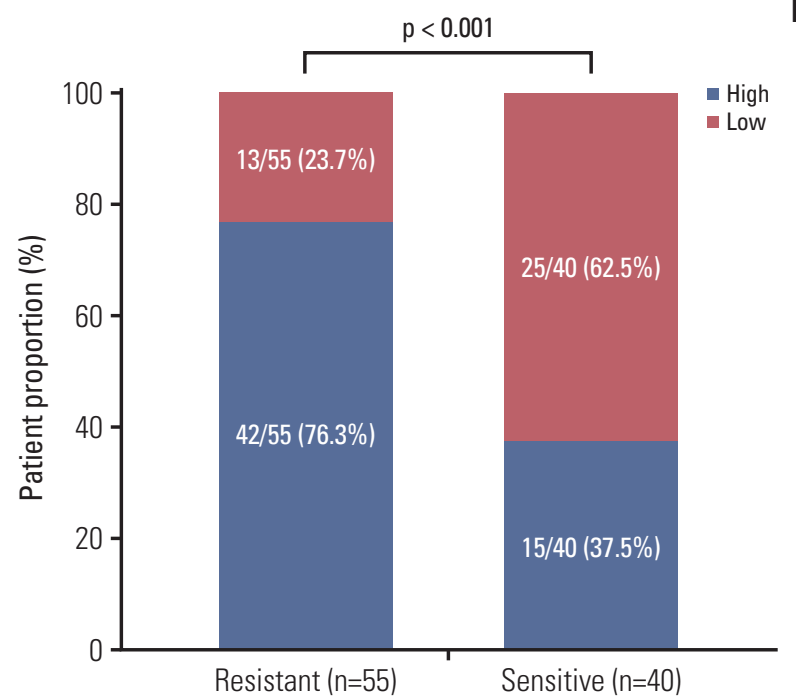

Fig. 2. Results of immunohistochemical (IHC) analysis. (A) Median IHC score of the resistant group was greater than that of the sensitive group ( 8 vs. 3, p=0.005). (B) Patients with high-level C-C motif chemokine receptor 6 expression were more common in the resistant group than in the sensitive group $(76.3 \%$ vs. $37.5 \%, \mathrm{p}<0.001)$.

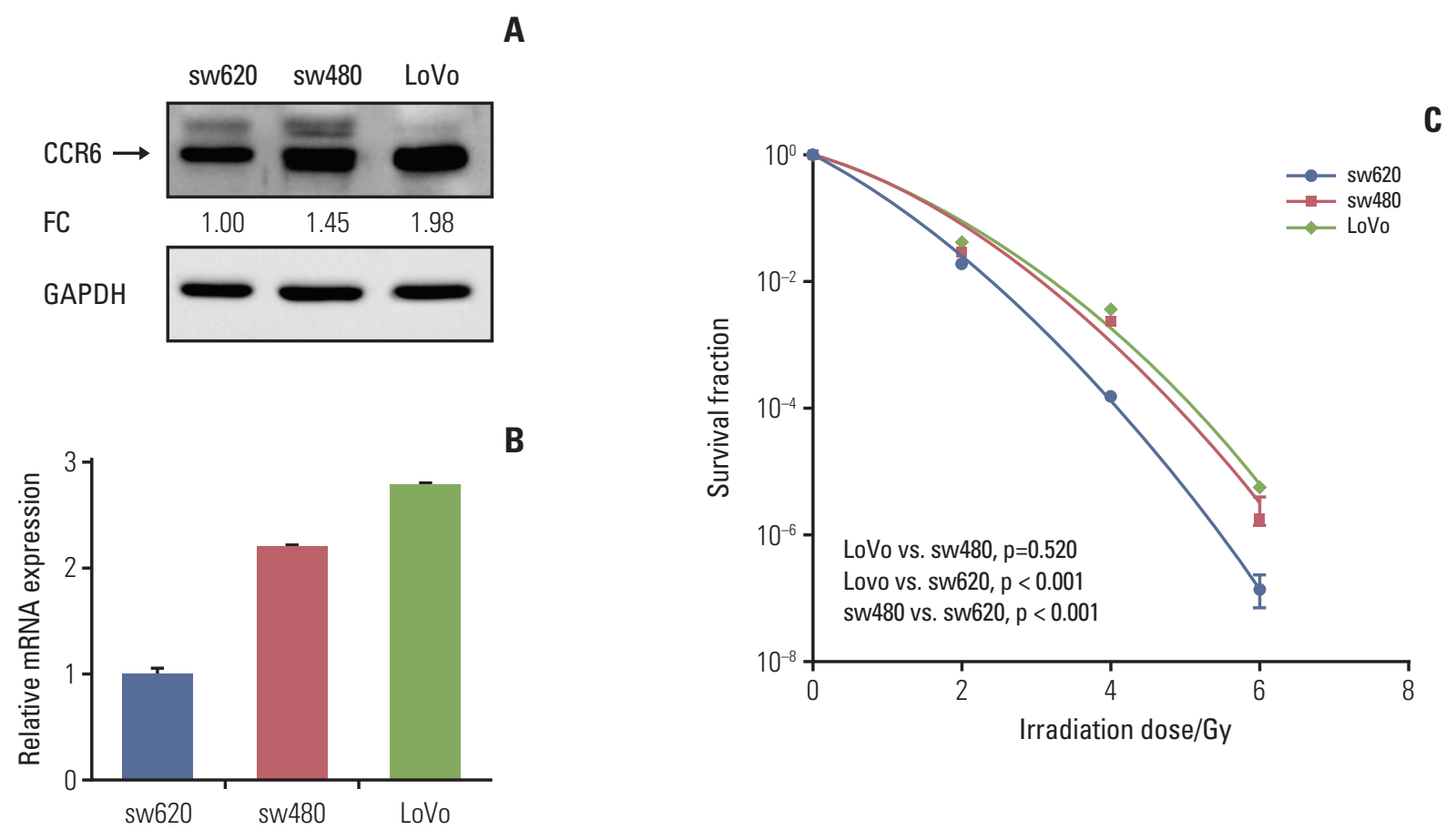

Fig. 3. Colorectal cancer (CRC) cell lines with high C-C motif chemokine receptor 6 (CCR6) expression were resistant to ionizing radiation. (A) Western blot analysis on CCR6 expression in the sw620, the sw480 and the LoVo cell lines. The reference cell line to calculate the fold change (FC) was the sw620 cell line. (B) Real-time polymerase chain reaction analysis on CCR6 expression in the CRC cell lines described in panel A. (C) Postirradiation survival curves of the CRC cell lines described in panel A. The sw480 and LoVo cells which presented higher CCR6 expression appeared to be more resistant to ionizing radiation. GAPDH, glyceraldehyde 3-phosphate dehydrogenase. 
Table 1. Parameters of survival curves for different colorectal cancer cells

\begin{tabular}{|c|c|c|c|}
\hline Cell line & $\alpha(/ G y)$ & $\beta\left(/ G y^{2}\right)$ & SF2 $(\%)$ \\
\hline LoVo & 0.363 & 0.083 & 24.6 \\
\hline sw480 & 0.369 & 0.091 & 22.8 \\
\hline sw620 & 0.641 & 0.084 & 17.6 \\
\hline LoVo-nc & 0.257 & 0.101 & 26.7 \\
\hline LoVo-si & 0.546 & 0.106 & 16.4 \\
\hline
\end{tabular}

SF2, survival fraction at 2 Gy; LoVo-nc, LoVo cells transfected with negative control; LoVo-si, siRNA-transfected LoVo cells.

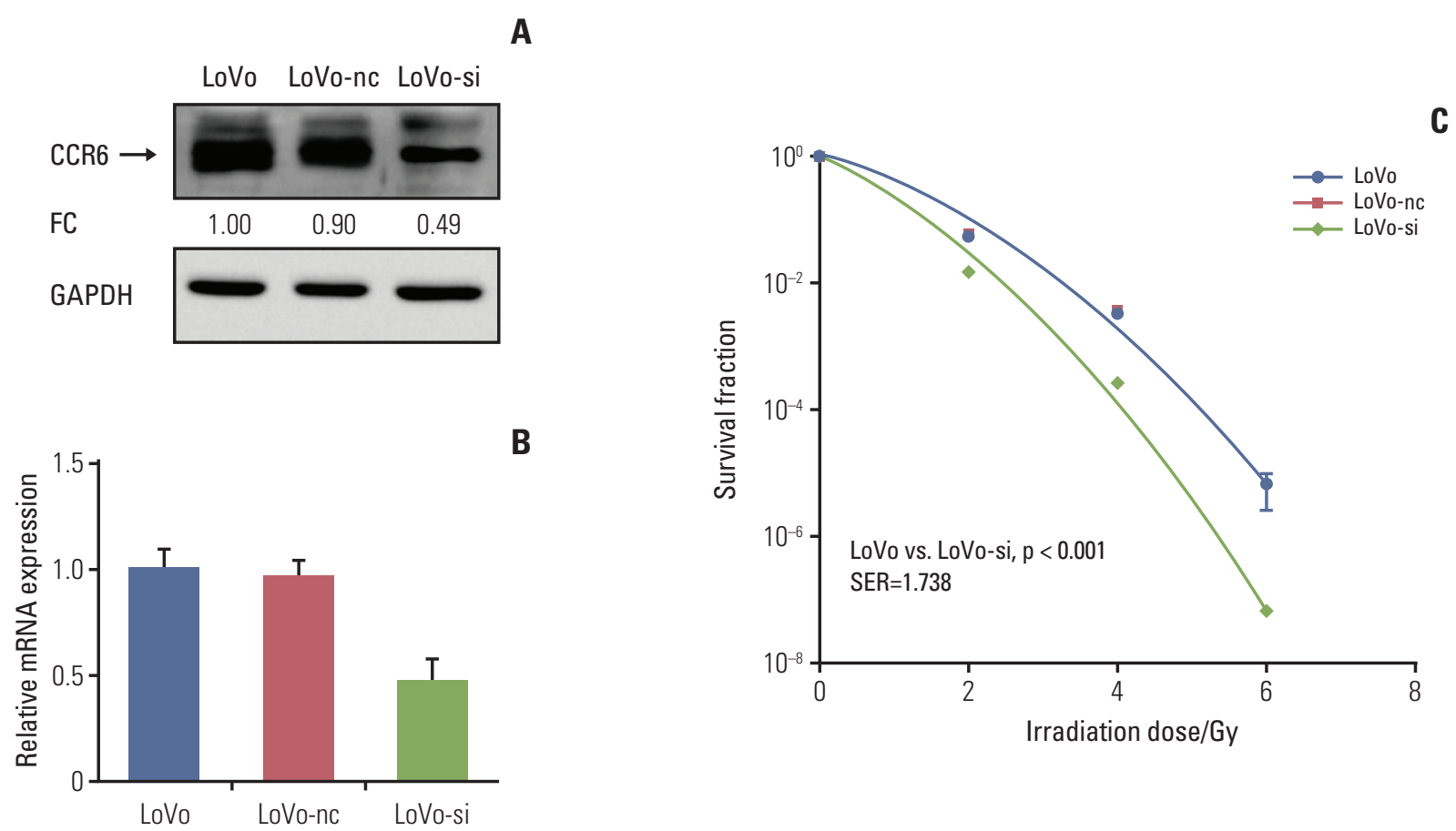

Fig. 4. Inhibition of C-C motif chemokine receptor 6 (CCR6) expression improved radioresistance of colorectal cancer (CRC) cells. (A) Western blot analysis on CCR6 expression in the LoVo cells, the LoVo cells transfected with negative control (LoVonc), and the siRNA-transfected LoVo cells (LoVo-si). The reference cell line to calculate the fold change (FC) was the LoVo cell line. (B) Real-time polymerase chain reaction analysis on CCR6 expression in the CRC cell lines described in panel A. (C) Postirradiation survival curves of the CRC cell lines described in panel A. The LoVo-si cells was more sensitive to ionizing radiation than the LoVo cells. The sensitization enhancement ratio (SER) was 1.738 ( $\mathrm{p}<0.001)$. GAPDH, glyceraldehyde 3-phosphate dehydrogenase.

good responders.

Taking these data together, CCR6 expression was inversely correlated with RT effects of the RC patients.

\section{CRC cells with high CCR6 expression was resistant to irradiation}

To know the exact role of CCR6 in radioresistance of $\mathrm{RC}$, CCR6 expression level was tested in different CRC cell lines. The results of western blot and RT-PCR analyses were showed as Fig. 3A and B, respectively. The sw480 and LoVo 


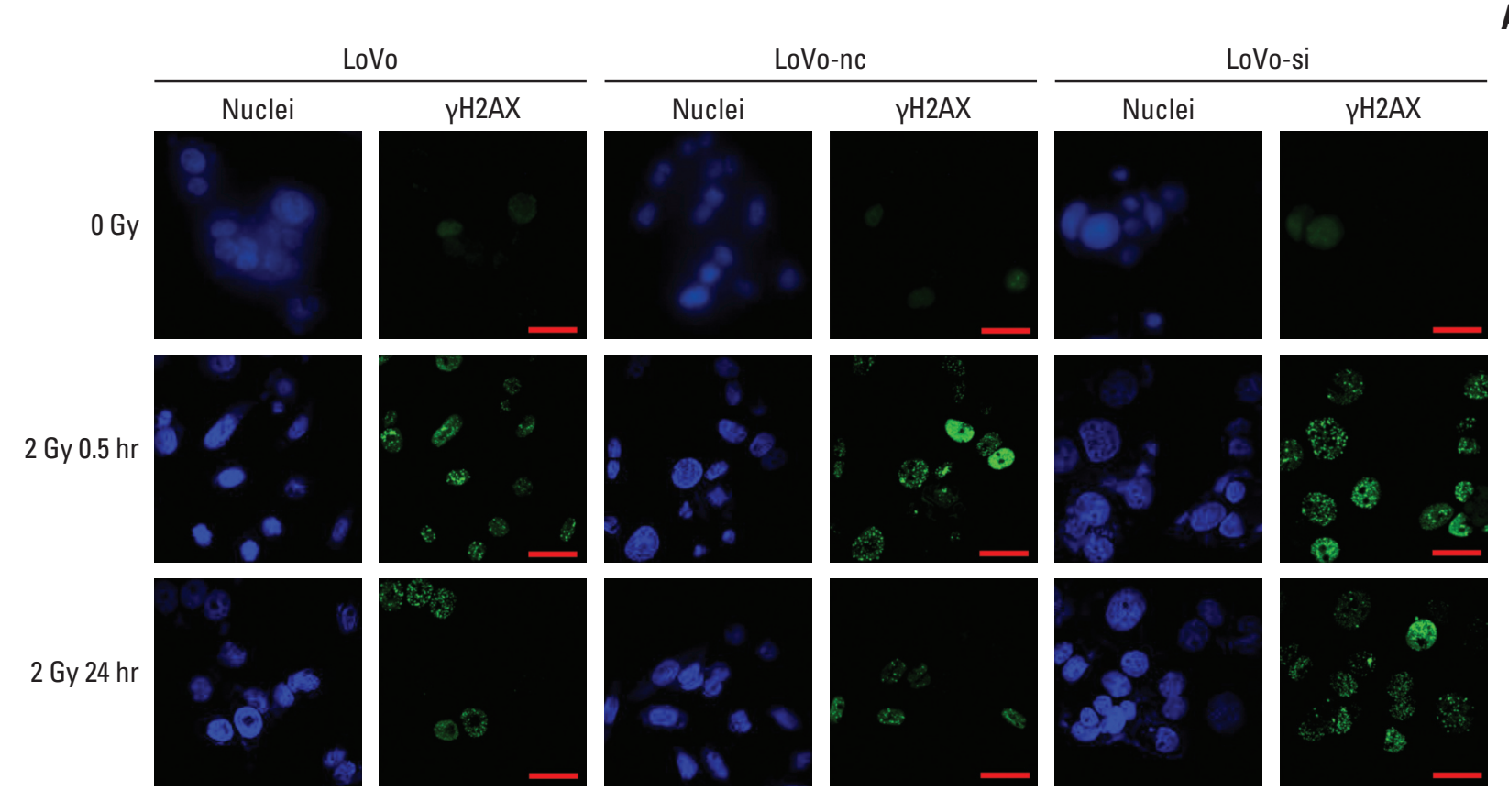

A

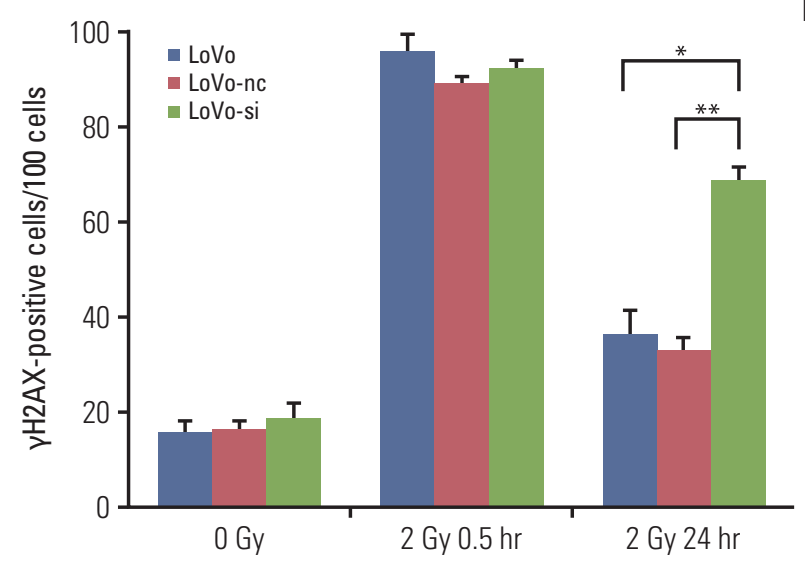

Fig. 5. C-C motif chemokine receptor 6 knockdown resulted in retardation of postirradiation DNA damage repair in colorectal cancer (CRC) cells. (A) Immunofluorescent staining of nuclei and $\gamma \mathrm{H} 2 \mathrm{AX}$ in LoVo, LoVo cells transfected with negative control (LoVo-nc), and siRNA-transfected LoVo cells (LoVo-si) cell lines ( $\times 400)$. Each cell line was stained at 0 Gy, and at 30 minutes and 24 hours after 2-Gy irradiation. Scale bars $=30 \mu \mathrm{m}$. (B) Quantification of $\gamma \mathrm{H} 2 \mathrm{AX}$-positive cells (in number per 100 cells). Similar numbers of $\gamma \mathrm{H} 2 \mathrm{AX}$-positive cells were seen among the three CRC cell lines described in panel A, at either 0 Gy or 30 minutes after 2-Gy irradiation. However, more $\gamma \mathrm{H} 2 \mathrm{AX}$-positive cells were seen in the LoVo-si cell line than in the LoVo-nc and the LoVo cells lines at 24 hours after 2-Gy irradiation $\left({ }^{*} \mathrm{p}<0.05,{ }^{* *} \mathrm{p}<0.01\right)$. 
cells presented a relatively high level of CCR6 expression. Contrarily, there was a relatively low level of CCR6 expression in the sw620 cells. The clonogenic survival assay indicated that CRC cell lines were arranged in descending order of radioresistance as following: $\mathrm{LoVo} \approx \mathrm{Sw} 480>\mathrm{sw} 620$ (Fig. 3C). The SF2 of the three cell lines were $24.6 \%, 22.8 \%$, and $17.8 \%$, respectively (Table 1 ). Thus, there was an up-regulation of CCR6 expression in the cell lines relatively resistant to IR. It indicated that CCR6 might participate in regulation of $\mathrm{RC}$ radiosensitivity.

\section{CCR6 knockdown sensitized CRC cells to irradiation}

In vitro silencing targeting CCR6 was performed in the LoVo cells which expressed relatively high level of CCR6 and had the greatest SF2, to validate the impact of CCR6 on radiosensitivity. The silencing efficiency was confirmed by western blot and RT-PCR analyses (Fig. 4A and B). The clonogenic survival assay showed that the siRNA-transfected LoVo cells (LoVo-si) were more sensitive to IR, compared with the LoVo cells. The SER was 1.738 ( $\mathrm{p}<0.001$ ) (Fig. 4C). No radiosensitizing effect was seen in the LoVo cells transfected with negative control (LoVo-nc). The results of RNA interference further supported the ability of CCR6 in modulating the sensitivity of RC to IR.

\section{Postirradiation DDR in CRC cells was retarded by CCR6 knockdown}

The $\gamma \mathrm{H} 2 \mathrm{AX}$ is known to recognize the sites of DNA damage and initiate the DDR procedure. Therefore, the $\gamma \mathrm{H} 2 \mathrm{AX}$ has now been used as a practical molecular marker reflecting the existence of DNA damage [5,21]. Quantification of $\gamma \mathrm{H} 2 \mathrm{AX}$-positive cells (in number per 100 cells) was conducted to decide the DDR abilities of CRC cell lines with different levels of CCR6 expression. Through IF analysis, the $\gamma \mathrm{H} 2 \mathrm{AX}$-positive cells appeared to be similar among the LoVo, the LoVo-nc and the LoVo-si cell lines, at baseline and 30 minutes after $2 \mathrm{~Gy}$ irradiation. It indicated that irradiation caused similar DNA damage among the three cell lines. However, at 24 hours after 2-Gy irradiation, residual $\gamma \mathrm{H} 2 \mathrm{AX}$ positive cells were significantly less in the LoVo ( $35.6 \pm 6.3$ vs. $68.0 \pm 4.9, \mathrm{p}=0.022)$ and the LoVo-nc (32.0 \pm 3.5 vs. $68.0 \pm 4.9$, $\mathrm{p}=0.009)$ cell lines than in the LoVo-si cell line (Fig. 5). In other words, the DDR was retarded and more DNA damage remained in the LoVo-si cell line, in which CCR6 was knockdown. It implied that CCR6 might regulate radioresistance by affecting efficiency of IR-induced DDR.

\section{Discussion}

The biological functions of CCR6 in metastasis and its predicting value in long-term outcome have been demonstrated in a series of studies. Chin et al. [22] showed in an in vitro study that activation of CCR6 could promote migration of CRC cells. And Kapur et al. [9] and Ghadjar et al. [8] further attained clinical evidences that high level of CCR6 expression would facilitate the distant metastasis of CRC, and was able to predict a poorer survival independently. Since therapeutic effect of RT is also so important for patients with locally advanced RC, impact of CCR6 on radiosensitivity of RC was explored in this study. It was found in RC patients that there was an inverse correlation between CCR6 expression and RT effect. Both the RNA-seq and the IHC analyses indicated that the lowlier CCR6 was expressed, the worse TRG would be got. Similar results were seen in CRC cell lines. Clonogenic survival assay after irradiation revealed that CCR6 expression was upregulated in cell lines (sw480 and LoVo) relatively resistant to IR. And on the contrary, down-regulation of CCR6 expression was seen in sw620, a radiosensitive cell line. Moreover, when CCR6 expression was interfered through siRNA, the LoVo cells would become sensitive to IR as well. These results all suggested that CCR6 might be a predicting biomarker of RT effect and a potential therapeutic target for reversing radioresistance. Prior to our study, there was no study focusing on the correlation between CCR6 expression and RC radiosensitivity. So, we are convinced the results might be informative for development of RT-sensitizing treatments and selection of individualized therapeutic strategies. Considering the proven influences of CCR6 on distant metastasis of CRC, the results of this study conferred more values on this biomarker, in improving prognosis of the RC patients.

The IR causes cell death mainly through double strand break of DNA. After irradiation, a tumor cell will survive if DDR is completed successfully. Or the programmed death will start instead. Therefore, the intrinsic resistance caused by DDR is acknowledged as the most critical mechanism of cellular radioresistance $[5,23]$. The mammalian DDR procedure is a phosphorylation signaling cascade. The first step is the phosphorylation on serine 139 of H2AX by ATM. The $\gamma \mathrm{H} 2 \mathrm{AX}$ will be dephosphorylated soon after DDR restores chromatin integrity $[21,24,25]$. Hence, the quantity of $\gamma \mathrm{H} 2 \mathrm{AX}$ is positively correlated with the number of DNA damage. We evaluated DDR abilities of different CRC cell lines after irradiation through quantifying the $\gamma \mathrm{H} 2 \mathrm{AX}$-positive cells in this study. At 30 minutes after irradiation, an obviously increase of $\gamma \mathrm{H} 2 \mathrm{AX}$-positive cells was seen in all the three tested cell lines, compared with the baseline. It indicated that DDR started as expected. And no difference in positive cell 
numbers was seen among the three cell lines, either before irradiation or at 30 minutes after irradiation. That was to say, similar amount of DNA damage was caused among the three cell lines. The numbers of $\gamma \mathrm{H} 2 \mathrm{AX}$-positive cells then decreased at 24 hours after irradiation, indicating the gradual completion of DDR. But comparison among the three cell lines revealed that there was a delay of $\gamma \mathrm{H} 2 \mathrm{AX}$ clearance in the LoVo-si cell, unlike the LoVo and the LoVo-nc cells. It could be easily inferred that DDR was disrupted when CCR6 expression was interfered. Namely, CCR6 might play its part in regulation of radiosensitivity through affecting DDR efficiency.

Up to now, the exact mechanisms through which CCR6 regulates DDR remain uncertain. This regulation might lie on the Akt and the ERK pathways which could be activated by the combination of CCR6 and its sole ligand, CCL20 [11]. The activated Akt has been reported to stabilize the ubiquitin-conjugating enzyme E2S, which will subsequently recruit the Ku70, a key molecule in DDR process [12]. The Akt could also upregulate the expression of Rad51. The latter is a molecule participating in the DDR directly [13]. For ERK, it has been showed in a study of Marampon et al. [14] to upregulate the expression of the regulatory and catalytic subunits of the DDR complex, DNA-PK, after its activation. In addition, the Akt and the ERK have been reported to inhibit the programmed cell death through p53 and mammalian target of rapamycin pathways, which are activated when DDR fails to complete [26-28]. Further studies are in need to figure out whether CCR6 controls the DDR and radiosensitivity of CRC cells through these pathways.

More and more biological functions of the tumor cells, including resistance to IR or chemotherapy agents, has been discovered to be determined by the TME [6,29]. Chemokines receptors are important components linking the TME and the tumor cells. Through the pathway enrichment analysis on our RNA-seq data, the chemokine-mediated signaling path- way was showed as the fourth enriched pathways $(\mathrm{p}<0.001)$ involved in RC radioresistance. Actually, before our study, another chemokine receptor, CXCR4, has been proven to regulate radiosensitivity of glioblastoma and uterine cervical cancer [30]. The results of our study added knowledge to this field and might help radiation oncologists to design further researches to explore and interfere the relationship between TME and the radioresistence of malignant tumors. Furthermore, the modalities blocking the interaction between chemokines and their receptors have gradually been developed [29]. The chemokine receptors are expected to be practical targets for radiosensitization treatment.

In conclusion, this study demonstrated that CCR6 overexpression contributed to a poorer RT effect in RC patients and radioresistance in CRC cells. And the results also implied that the CCR6 might enhance radioresistance through more effective DDR. Thus, inhibition of CCR6 expression might be a therapeutic strategy to sensitize RC to RT.

\section{Electronic Supplementary Material}

Supplementary materials are available at Cancer Research and Treatment website (http:// www.e-crt.org).

\section{Conflicts of Interest}

Conflict of interest relevant to this article was not reported.

\section{Acknowledgments}

We are grateful to Dr. Jia-bin Lu and Dr. Jie-wei Chen (Department of Pathology, Sun Yat-sen University Cancer Center, Guangzhou, China) for their technical help in the assessment of the patients' tissue specimens.

This study was supported by the National Natural Science Foundation of China (Grant Number: 81672987).

\section{References}

1. Chen W, Zheng R, Baade PD, Zhang S, Zeng H, Bray F, et al. Cancer statistics in China, 2015. CA Cancer J Clin. 2016;66: 115-32.

2. Park HJ, Cho S, Kim Y. Patterns of rectal cancer radiotherapy adopting evidence-based medicine: an analysis of the national database from 2005 to 2016. Cancer Res Treat. 2018;50:975-83.

3. Fokas E, Liersch T, Fietkau R, Hohenberger W, Beissbarth T, Hess C, et al. Tumor regression grading after preoperative chemoradiotherapy for locally advanced rectal carcinoma revisited: updated results of the $\mathrm{CAO} / \mathrm{ARO} / \mathrm{AIO}-94$ trial.
J Clin Oncol. 2014;32:1554-62.

4. Trakarnsanga A, Gonen M, Shia J, Nash GM, Temple LK, Guillem JG, et al. Comparison of tumor regression grade systems for locally advanced rectal cancer after multimodality treatment. J Natl Cancer Inst. 2014;106:dju248.

5. Morgan MA, Lawrence TS. Molecular pathways: overcoming radiation resistance by targeting DNA damage response pathways. Clin Cancer Res. 2015;21:2898-904.

6. Barker HE, Paget JT, Khan AA, Harrington KJ. The tumour microenvironment after radiotherapy: mechanisms of resist- 
ance and recurrence. Nat Rev Cancer. 2015;15:409-25.

7. Zlotnik A, Burkhardt AM, Homey B. Homeostatic chemokine receptors and organ-specific metastasis. Nat Rev Immunol. 2011;11:597-606.

8. Ghadjar P, Coupland SE, Na IK, Noutsias M, Letsch A, Stroux $\mathrm{A}$, et al. Chemokine receptor CCR6 expression level and liver metastases in colorectal cancer. J Clin Oncol. 2006;24:1910-6.

9. Kapur N, Mir H, Clark CE 3rd, Krishnamurti U, Beech DJ, Lillard JW, et al. CCR6 expression in colon cancer is associated with advanced disease and supports epithelial-to-mesenchymal transition. Br J Cancer. 2016;114:1343-51.

10. Nandi B, Pai C, Huang Q, Prabhala RH, Munshi NC, Gold JS. CCR6, the sole receptor for the chemokine CCL20, promotes spontaneous intestinal tumorigenesis. PLoS One. 2014;9: e97566.

11. Frick VO, Rubie C, Keilholz U, Ghadjar P. Chemokine/ chemokine receptor pair CCL20/CCR6 in human colorectal malignancy: an overview. World J Gastroenterol. 2016;22: 833-41.

12. Hu L, Li X, Liu Q, Xu J, Ge H, Wang Z, et al. UBE2S, a novel substrate of Akt1, associates with $\mathrm{Ku} 70$ and regulates DNA repair and glioblastoma multiforme resistance to chemotherapy. Oncogene. 2017;36:1145-56.

13. Ko JC, Chen JC, Wang TJ, Zheng HY, Chen WC, Chang PY, et al. Astaxanthin down-regulates Rad51 expression via inactivation of AKT kinase to enhance mitomycin C-induced cytotoxicity in human non-small cell lung cancer cells. Biochem Pharmacol. 2016;105:91-100.

14. Marampon F, Gravina GL, Zani BM, Popov VM, Fratticci A, Cerasani M, et al. Hypoxia sustains glioblastoma radioresistance through ERKs / DNA-PKcs / HIF-1alpha functional interplay. Int J Oncol. 2014;44:2121-31.

15. Mandric I, Temate-Tiagueu Y, Shcheglova T, Al Seesi S, Zelikovsky A, Mandoiu II. Fast bootstrapping-based estimation of confidence intervals of expression levels and differential expression from RNA-Seq data. Bioinformatics. 2017;33: 3302-4.

16. Tripathi S, Pohl MO, Zhou Y, Rodriguez-Frandsen A, Wang G, Stein DA, et al. Meta- and orthogonal integration of influenza "OMICs" data defines a role for UBR4 in virus budding. Cell Host Microbe. 2015;18:723-35.

17. Chen DL, Chen LZ, Lu YX, Zhang DS, Zeng ZL, Pan ZZ, et al. Long noncoding RNA XIST expedites metastasis and modulates epithelial-mesenchymal transition in colorectal cancer. Cell Death Dis. 2017;8:e3011.

18. Cerda MB, Lloyd R, Batalla M, Giannoni F, Casal M, Policastro
L. Silencing peroxiredoxin-2 sensitizes human colorectal cancer cells to ionizing radiation and oxaliplatin. Cancer Lett. 2017;388:312-9.

19. Untergasser A, Cutcutache I, Koressaar T, Ye J, Faircloth BC, Remm M, et al. Primer3: new capabilities and interfaces. Nucleic Acids Res. 2012;40:e115.

20. Tu Z, Xu B, Qu C, Tao Y, Chen C, Hua W, et al. BRCC3 acts as a prognostic marker in nasopharyngeal carcinoma patients treated with radiotherapy and mediates radiation resistance in vitro. Radiat Oncol. 2015;10:123.

21. Kleiner RE, Verma P, Molloy KR, Chait BT, Kapoor TM. Chemical proteomics reveals a gammaH2AX-53BP1 interaction in the DNA damage response. Nat Chem Biol. 2015;11: $807-14$

22. Chin CC, Chen CN, Kuo HC, Shi CS, Hsieh MC, Kuo YH, et al. Interleukin-17 induces CC chemokine receptor 6 expression and cell migration in colorectal cancer cells. J Cell Physiol. 2015;230:1430-7.

23. Maier P, Hartmann L, Wenz F, Herskind C. Cellular pathways in response to ionizing radiation and their targetability for tumor radiosensitization. Int J Mol Sci. 2016;17:E102.

24. Lee JS. Activation of ATM-dependent DNA damage signal pathway by a histone deacetylase inhibitor, trichostatin A. Cancer Res Treat. 2007;39:125-30.

25. Moon SH, Lin L, Zhang X, Nguyen TA, Darlington Y, Waldman AS, et al. Wild-type p53-induced phosphatase 1 dephosphorylates histone variant gamma-H2AX and suppresses DNA double strand break repair. J Biol Chem. 2010;285: 12935-47.

26. Zhu Y, Dai B, Zhang H, Shi G, Shen Y, Ye D. Long non-coding RNA LOC572558 inhibits bladder cancer cell proliferation and tumor growth by regulating the AKT-MDM2-p53 signaling axis. Cancer Lett. 2016;380:369-74.

27. Mendoza MC, Er EE, Blenis J. The Ras-ERK and PI3K-mTOR pathways: cross-talk and compensation. Trends Biochem Sci. 2011;36:320-8.

28. Sato A, Sunayama J, Matsuda K, Seino S, Suzuki K, Watanabe $\mathrm{E}$, et al. MEK-ERK signaling dictates DNA-repair gene MGMT expression and temozolomide resistance of stem-like glioblastoma cells via the MDM2-p53 axis. Stem Cells. 2011;29: 1942-51.

29. Sun Y. Tumor microenvironment and cancer therapy resistance. Cancer Lett. 2016;380:205-15.

30. Trautmann F, Cojoc M, Kurth I, Melin N, Bouchez LC, Dubrovska A, et al. CXCR4 as biomarker for radioresistant cancer stem cells. Int J Radiat Biol. 2014;90:687-99. 